\title{
Amplification of short pulses from a mode-locked diode laser in an ytterbium-doped fiber
}

\author{
M.G. Hekelaar, B. Adhimoolam, P. Gross, I.D. Lindsay, and K.-J. Boller \\ Dept. of Applied Physics, University of Twente, The Netherlands \\ p.gross@utwente.nl
}

\begin{abstract}
We report the first mode-locked diode laser at $1.04 \mu \mathrm{m}$ with subsequent amplification of the pulses in an ytterbium-doped fiber amplifier. The generated pulses have a pulse duration of $70 \mathrm{ps}$ and peak power of $50 \mathrm{~W}$.
\end{abstract}

keywords semiconductor laser, mode locking, fiber amplifier

Introduction Over the last decade, there has been considerable interest in powerful mode-locked light sources in the wavelength range around $1 \mu \mathrm{m}$. Such sources are ideal for sensitive environmental monitoring and for test and measurement purposes. In particular, they offer the possibility to reach further into the infrared spectral region by efficient wavelength conversion processes, e.g. by synchronously pumping optical parametric oscillators (OPOs) $[1,2]$.

Interest in the $1 \mu \mathrm{m}$ region has been enhanced by recent developments in cladding pumped ytterbium-doped silica fiber lasers and amplifiers. Offering a broad gain bandwidth and a high pump conversion efficiency, they form an almost ideal gain medium for the generation and amplification of wavelength-flexible and short pulses in the spectral region between 1000 and $1100 \mathrm{~nm}$. Short pulse generation from Yb-doped fiber lasers, also followed by $\mathrm{Yb}$-fiber amplifiers, has been demonstrated [3-5], however, due to the high dispersion in such a system, rather complex cavities are required. Additionally, the extended fiber lengths lead to an inherently low repetition rate (tens of $\mathrm{MHz}$ ) such that synchronous pumping of e.g. OPOs requires larger cavity lengths.

Mode-locked semiconductor lasers are a highly attractive alternative to mode-locked bulk and fiber solid state laser oscillators. Such diodes offer high $(\mathrm{GHz})$ repetition rates for e.g. synchronous pumping of compact OPOs. The electronically controllable repetition rate can be employed for rapid OPO tuning [6], while moderate pulse duration in the picosecond range ensure efficient conversion in long non-linear crystals. In addition, the limited spectral bandwidth of these pulses yields a spectral resolution suitable for spectroscopic detection schemes in ambient pressure broadened molecular species. Surprisingly, despite the potential as seed sources for Yb-doped fiber amplifiers, there seems to be only a single experimental demonstration of a mode-locked laser at around $1 \mu \mathrm{m}$ wavelength [7]. In that case an internal absorber was implemented to generate pulses of around $1 \mathrm{ps}$ duration. Nevertheless, mode locking should be possible with a standard Fabry-Perot diode laser when anti-reflection coated and operated in an external cavity. Further there seems no demonstration of fiber amplification of such diodes.

Here we report what we believe is the first example of an actively mode locked diode laser near $1 \mu \mathrm{m}$ with subsequent amplification of the pulses in an ytterbium-doped fiber amplifier.

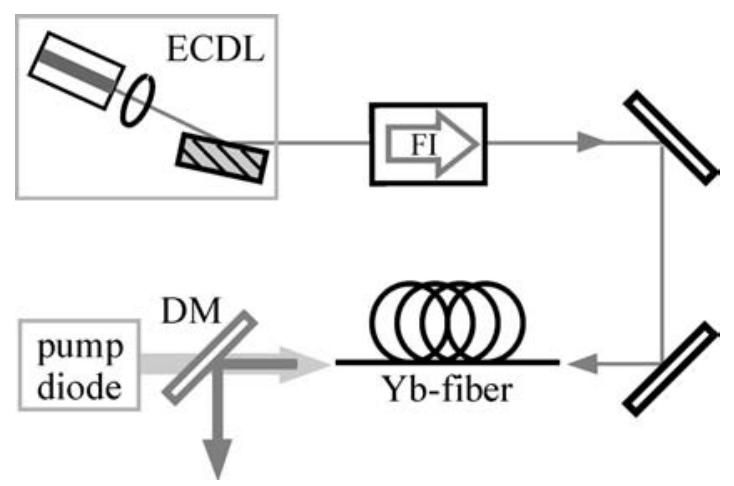

Fig. 1. Setup of the diode-oscillator fiber-amplifier system. ECDL, external cavity diode laser; FI, Faraday optical isolator; DM, dichroic mirror.

Experimental setup The experimental setup is shown in Fig. 1. The diode laser is a single-stripe InGaAs-GaAs diode with its output facet anti-reflection coated to a residual reflectivity specified as less than $10^{-5}$. To allow modulation of the driving current with frequencies in the GHz-range, we equipped the diode with an impedance matching circuit. The diode is connected via a bias-T to a dc biasing supply and 
an rf source (Anritsu 69147A synthesizer and a Trontech P1020-33 amplifier). The diode laser is mounted in an external grating cavity in Littrow configuration for coarse wavelength selection [8].

After transmission through a $60-\mathrm{dB}$ optical isolator, the diode laser emission is amplified in a 36-m long ytterbium-doped double-clad fiber with an active core diameter of $10 \mu \mathrm{m}$ and a NA of 0.07 [9]. The D-shaped inner cladding with $400-\mu \mathrm{m}$ diameter and a NA of 0.38 is end-pumped by a diode laser with up to $25 \mathrm{~W}$ at $976 \mathrm{~nm}$.

The duration of the pulses generated by the diode laser is measured with an autocorrelator based on a pair of rotating mirrors [10]. A rather long (1 $\mathrm{cm}) \mathrm{BBO}$ frequency-doubling crystal is used for increased conversion efficiency. This enables a photomultiplier-based detection of the second harmonic also with the low $(\mathrm{mW})$ fundamental average power levels provided by the diode laser.

Results For mode locking of the external cavity diode laser we use an rf modulated drive current superimposed on a dc drive current. The cavity length is adjusted to $107 \mathrm{~mm}$ to match the rf drive frequency of $1.4 \mathrm{GHz}$. In the present experiment the wavelength is set to $1040 \mathrm{~nm}$ via angle tuning of the grating. With a dc current of $60 \mathrm{~mA}$ and an rf power of $29 \mathrm{dBm}$, the diode laser generates $7 \mathrm{~mW}$ of average output power with a measured spectral bandwidth of less than $0.1 \mathrm{~nm}$. The autocorrelation trace, which is shown in Fig. 2a, is consistent with a pulse of 70 ps duration and with a nonlinear chirp.

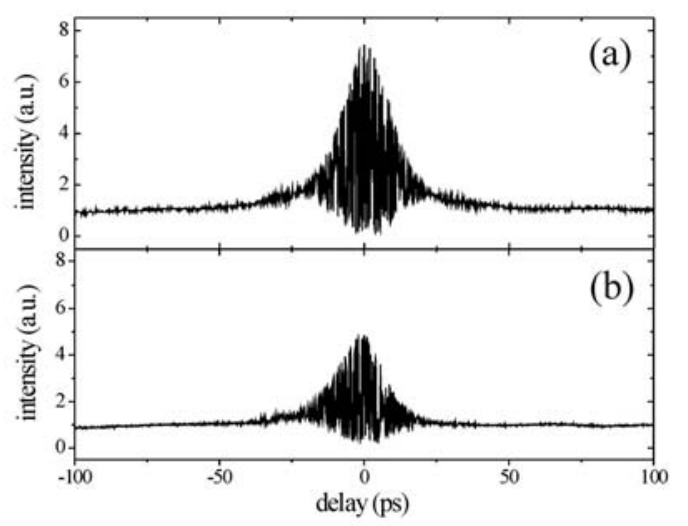

Fig. 2. Autocorrelation trace of the diode laser pulses, before (a) and after (b) amplification.

The pulses from the diode laser are injected into the fiber amplifier. With a pump power of $19 \mathrm{~W}$, limited by self-pulsing due to the unsaturated gain, the diode laser pulses are amplified to more than $5 \mathrm{~W}$ of average power. The autocorrelation trace of the amplified pulses in Fig. $2 b$ shows that the pulse duration of $70 \mathrm{ps}$ is maintained during amplification. However, the peak-to-background ratio in the autocorrelation trace has decreased, which can be attributed to an additional linear chirp obtained in the fiber.

In conclusion, we present the first amplification of ultra-short pulses from a mode-locked 1040-nm diode laser in an ytterbium-doped fiber amplifier. We have generated pulses with a duration of $70 \mathrm{ps}$ and with $5 \mathrm{~W}$ average power, corresponding to $50 \mathrm{~W}$ peak power. Given the narrow spectral bandwidth of $0.1 \mathrm{~nm}$ and the potential of broad wavelength tunability, but also because of the $\mathrm{GHz}$ repetition rate such diode-oscillator fiber-amplifier systems are quite attractive for non-linear frequency conversion in compact synchronously pumped OPOs.

\section{References}

[1] A. Robertson, M.E. Klein, M.A. Tremont, K.-J. Boller, and R. Wallenstein, Opt. Lett. 25, 657 (2000)

[2] S. Lecomte et al., Opt. Lett. 30, 290 (2004)

[3] A. Hideur, T. Chartier, M. Brunel, S. Louis, C. Özkul, and F. Sanchez, Appl. Phys. Lett. 79, 3389 (2001)

[4] O.G. Okhotnikov, L. Gomes, N. Xiang, T. Jouhti, and A.B. Grudinin, Opt. Lett. 28, 1522 (2003)

[5] A. Malinowski, A. Piper, J.H.V. Price, K. Furusawa, Y. Jeong, J. Nilsson, and D.J. Richardson, Opt. Lett. 29, 2073 (2004)

[6] M.E. Klein, A. Robertson, M.A. Tremont, R. Wallenstein, and K.-J. Boller, Appl. Phys. B 73, 1 (2000)

[7] M.J. Brennan, A.J. Budz, B.J. Robinson, P. Mascher, and H.K. Haugen, IEEE Photon. Techn. Lett. 16, 1798 (2004)

[8] T.W. Hänsch, Appl. Optics 11, 895 (1972)

[9] M.E. Klein, P. Gross, K.-J. Boller, M. Auerbach, P. Wessels, and C. Fallnich, Opt. Lett. 28, 920 (2003)

[10] D.M. Riffe and A.J. Sabbah, Rev. Sci. Instr. 69, 3099 (1998) 